\title{
THE LEAST ACTION PRINCIPLE AND THE RELATED CONCEPT OF GENERALIZED FLOWS FOR INCOMPRESSIBLE PERFECT FLUIDS
}

\author{
YANN BRENIER
}

\section{INTRODUCTION}

Let us consider the motion of a perfect incompressible fluid inside a closed bounded domain $X$ in the Euclidian space $\mathbf{R}^{d}$. The fluid is not necessarily homogeneous, which means that the initial mass density $\rho_{0}(x)$ may depend on the space variable $x$. If the external forces derive from a potential $U(t, x)$ (where $t$ is the time variable), then the motion is described in terms of the density field $\rho(t, x)$, the velocity field $v(t, x)$, and the pressure field $p(t, x)$ by the following equations:

$$
\begin{gathered}
\operatorname{div} v=0 \quad \text { (incompressibility condition), } \\
\partial_{t} \rho+v \cdot \operatorname{grad} \rho=0 \quad \text { (conservation of mass), } \\
\rho\left\{\partial_{t} v+v \cdot \operatorname{grad} v+\operatorname{grad} U\right\}+\operatorname{grad} p=0 \quad \text { (Newton's law). }
\end{gathered}
$$

The initial and boundary conditions are

$$
\begin{gathered}
v(t=0, x)=v_{0}(x), \quad \rho(t=0, x)=\rho_{0}(x), \\
v \cdot n=0 \text { along } \partial X
\end{gathered}
$$

where $n=$ outward normal (impermeability condition).

The pressure field is the Lagrange multiplier associated with the incompressibility condition (1.1) and needs neither boundary nor initial conditions. This system can be reformulated in terms of the flow map $g(t, x)$ defined on $\mathbf{R} \times X$, valued in $X$, and a solution to the ordinary differential system

$$
g(0, x)=x, \quad \partial_{t} g(t, x)=v(t, g(t, x)) .
$$

Received by the editors July 6, 1988 .

1980 Mathematics Subject Classification (1985 Revision). Primary 35D99, 76C99.

Key words and phrases. Euler equations, incompressible flows, continuous linear programming, path integrals, least action principle.

This work was completed while the author was visiting the University of California, Los Angeles. Research was supported by the NSF under grant DM585-03294 and the ONR under grant N0001486-K0691. 
For (1.2)-(1.4), we get

$$
\begin{gathered}
\rho(t, g(t, x))=\rho(t=0, x)=\rho_{0}(x), \\
\rho_{0}(x)\left\{\partial_{t}^{2} g(t, x)+\operatorname{grad} U(t, g(t, x))\right\}+\operatorname{grad} p(t, g(t, x))=0, \\
g(0, x)=x, \quad \partial_{t} g(0, x)=v_{0}(x) .
\end{gathered}
$$

If the velocity and the density fields, as well as $X$ and its boundary, are smooth enough, then the incompressibility and impermeability conditions (1.1)-(1.5) mean exactly that, for each time $t, g(t, \cdot)$ is a smooth diffeomorphism from $X$ into itself that preserves both orientation and volume. In other words,

$$
\text { for any } t, g(t, \cdot) \text { belongs to } G \text {, }
$$

where $G$ is the set of all smooth orientation and volume preserving mappings from $X$ into $X$,

$$
\begin{aligned}
G=\{\gamma: X \rightarrow X, & \text { diffeomorphism s.t. } \operatorname{det} D \gamma(x) \equiv 1\} \\
& (D \gamma(x) \text { denotes the Jacobian matrix of } \gamma \text { at } x) .
\end{aligned}
$$

Then the motion is entirely described by equations (1.8) and (1.9) and conditions (1.10) and (1.11). Behind these equations, as has been known for a long time [1], there is the Least Action Principle. Here the Action is the sum of the kinetic energy and the potential energy and is defined at each time $t$ by

$$
A(g, t)=\int_{X} \rho_{0}(x)\left\{\frac{1}{2}\left\|\partial_{t} g(t, x)\right\|^{2}-U(t, g(t, x))\right\} d x
$$

The Least Action Principle says that if $t_{1}-t_{0}>0$ is not too large, then

$$
\int_{t_{0}}^{t_{1}} A(g, t) d t \leq \int_{t_{0}}^{t_{1}} A(\gamma, t) d t
$$

holds for any flow map $\gamma$ such that

$$
\gamma(t, \cdot) \in G, \quad \text { for } t_{0} \leq t \leq t_{1}, \gamma\left(t_{0}, \cdot\right)=g\left(t_{0}, \cdot\right), \gamma\left(t_{1}, \cdot\right)=g\left(t_{1}, \cdot\right) .
$$

In other words, the Action integrand from $t_{0}$ to $t_{1}$ is minimal for $g$.

In this paper, instead of considering the initial value problem (1.8)-(1.11), we concentrate on the related minimization problem: given $t_{0}<t_{1}, g_{0}$ and $g_{1}$ in $G$, find a flow map $g$ such that

$$
g(t, \cdot) \in G, \quad \text { for } t_{0} \leq t \leq t_{1}, g\left(t_{0}, \cdot\right)=g_{0}, g\left(t_{1}, \cdot\right)=g_{1},
$$

and

$$
\int_{t_{0}}^{t_{1}} A(g, t) d t \text { is minimal. }
$$

This can be considered as a shortest path problem on $G$, the manifold of smooth orientation and volume preserving mappings from $X$ into itself. Since $G$ is a group under the composition rule, it is not a restriction to substitute the identity map for $g_{0}$ and $h=g_{1} \circ g_{0}^{-1}$ for $g_{1}$. In a similar way, one can substitute the time interval $[0, T]$ for $\left[t_{0}, t_{1}\right]$, where $T$ is a given strictly positive number. $h$ will be called the final configuration and $T$ the final time. Thus, let us consider 
The shortest path problem. Given $T>0$ and $h \in G$, find a flow map $t \rightarrow$ $g(t, \cdot) \in G, 0 \leq t \leq T$, that reaches the final configuration $h$ at time $T$ and minimizes the Action

$$
g \rightarrow \int_{0}^{T} \int_{X} \rho_{0}(x)\left\{\frac{1}{2}\left\|\partial_{t} g(t, x)\right\|^{2}-U(t, g(t, x))\right\} d x d t
$$

where $\rho_{0} \geq 0$ and $U$ are given.

From contributions by various authors [7], one can solve this problem when $h$ is very smooth ( $h$ belongs to some high order Sobolev space, say) and lies in a small neighborhood (related to some very strong topology) of the identity map. However, for arbitrary data, this minimization problem seems highly difficult since the quantity to be minimized does not involve any spatial derivatives of the flow map, while the incompressibility constraint is expressed in terms of the Jacobian determinant. Therefore, strong convergence of minimizing sequences cannot be obtained in classical ways. Moreover, the appropriate strong topologies for $G$ are totally unrelated to the metrics induced by the kinetic energy. The goal of this paper is to overcome these difficulties in the following two steps.

(i) Enlarge the framework with an adequate concept of generalized flows, in the spirit of L. C. Young's ideas on the calculus of variations [12], and prove the existence of a generalized solution to the shortest path problem.

(ii) Check that classical solutions cannot be missed in this new framework.

Before defining our favorite concept of generalized flow, let us review several possible generalizations of the shortest path problem. The first natural idea would be to substitute for the set $G$ a broader set of volume preserving mappings, for example,

$$
\begin{aligned}
& S=\{\gamma: X \rightarrow X, \text { if } Y \text { is a measurable subset of } X, \\
& \text { then } \left.\gamma^{-1}(Y) \text { is measurable and meas }\left(\gamma^{-1}(Y)\right)=\text { meas }(Y)\right\} .
\end{aligned}
$$

This definition is classical in ergodic theory [2]. An equivalent definition is

$$
\begin{aligned}
& S=\{\gamma: X \rightarrow X, \text { if } f \text { is a continuous function on } X, \\
& \text { then } \left.f \circ \gamma \text { is measurable and } \int_{X} f(\gamma(x)) d x=\int_{X} f(x) d x\right\} .
\end{aligned}
$$

These definitions make sense for any measure space $(X, d x)$ when $X$ is a compact set and $d x$ a positive Borel (Radon) measure. When $X$ is a closed bounded domain in $\mathbf{R}^{d}, G$ is obviously a subset of $S$, since for any $f$ in $C(X)$ and $\gamma$ in $G, f \circ \gamma$ is continuous and

$$
\begin{array}{r}
\int_{X} f(x) d x=\int_{X} f(\gamma(y)) \operatorname{det} D \gamma(y) d y=\int_{X} f(\gamma(y)) d y \\
\quad \text { (change of variable } x=\gamma(y)) .
\end{array}
$$

However, $S$ contains many more mappings than $G$. The 1-dimensional case $X=[-1,1]$ is striking since, then, $G$ has a single element (the identity map) 
while $S$ contains various mappings such as $\gamma(x)=x+1$ if $x<0, x-1$ if $x>0$, which is discontinuous, $\gamma(x)=-x$, which is orientation reversing, or $\gamma(x)=2 x+1$ if $x<0,1-2 x$ if $x>0$, which is not one-to-one. So $G$ and $S$ seem very different from each other. However, the gap between $G$ and $S$ is a specific property of the 1-dimensional case, and it is a reasonable conjecture to state that $S$ actually is the strong $L^{2}$ closure of $G$ if $d \geq 2$. We believe that such a result is probably available somewhere in the literature but, since it is not strictly necessary for our discussion, we will not try to prove it. In the shortest path problem, the quantity to be minimized looks like an $L^{2}$ norm with respect to the space variables. Therefore, it makes sense to substitute for $G$ what we believe to be its (strong) $L^{2}$-closure, that is, $S$. Then we get

The first generalization of the shortest path problem. Given $T>0$ and $h \in S$, find a measurable mapping $(t, x) \in[0, T] \times X \rightarrow g(t, x) \in X$ s.t.

$$
\begin{gathered}
g(0, x)=x, g(T, x)=h(x) \quad(\text { " } g \text { reaches } h \text { at time } T ") \\
\int_{X} f(g,(t, x)) d x=\int_{X} f(y) d y \quad \text { for any } t \in[0, T] \text { and } f \in C(X)
\end{gathered}
$$

(incompressibility),

$$
g \rightarrow \int_{0}^{T} \int_{X} \rho_{0}(x)\left\{\frac{1}{2}\left\|\partial_{t} g(t, x)\right\|^{2}-U(t, g(t, x))\right\} d x d t \text { is minimal. }
$$

This problem seems easier to solve than the original one, since the classical incompressibility condition

$$
\operatorname{det} D g(t, x) \equiv 1
$$

that involves, in a very nonlinear way, the space derivatives of the flow map is now replaced by condition (1.22), which makes sense even when the flow map is not continuous! However, it is still a nonlinear constraint. At this point of the discussion, it is highly questionable whether or not such a generalization is justified from a physical point of view. For example, the important property for the flow map to be, at least, one-to-one and orientation preserving is completely missed by this new framework. A reasonable answer to this question would be to prove, at least, that, whenever there is a classical solution to the original shortest path problem, it is automatically the solution to the generalized problem. Otherwise, the new framework would be worthless. Nevertheless, this is not our main concern at the moment, since it is not clear that even the generalized shortest path problem always has a solution! Indeed, as has been mentioned earlier, the action to be minimized does not involve any spatial derivative of the flow map and, therefore, there is not control of the amount of oscillation (in the space variables) that can be produced by the minimizing sequences. In some cases, for example,

$$
\begin{gathered}
X=[-1,1] \text { and } h(x)=-x, \quad \text { or more generally, } \\
X=[-1, d]^{d} \text { and } h(x)=\left(-x_{1}, x_{2}, \ldots, x_{d}\right)
\end{gathered}
$$


(not classical, since the final configuration $h$ is orientation reversing) oscillations occur, and (see §6) solutions must be looked for in a much larger class of flowmaps. This kind of trouble is typical in the calculus of variations, and this is why L. C. Young [12] introduced illuminating probabilistic concepts, known as Young's measures, to describe the behavior of oscillating minimizing sequences. These techniques have been popularized and extended to other fields by Tartar [11] and used in fluid mechanics, for both compressible [5] and incompressible [6] flows, through the concepts of measure-valued solutions. Unfortunately, this last concept is essentially Eulerian and not Lagrangian, in the sense that it is based on the description of the motion in terms of velocity fields (through equations (1.1)-(1.4)) rather than flowmaps (through (1.6)-(1.11)). Therefore, it does not seem suitable for the shortest path problem considered here, and a different (but consistent) concept will be introduced in this paper. Before describing what we believe to be the right probabilistic concept for our problem, let us first consider an intermediate generalization, which turns out to be inadequate but shows interesting features. Indeed, it is a natural probabilistic idea to replace the concept of volume preserving mappings by the one of doubly stochastic probability measures on the product space $X \times X$. This is classical in the literature devoted to the Monge-Kantorovitch problem [9] and has been recently used by the author [4] to define the arrangement of vector fields. A doubly stochastic probability measure on $X \times X$ is a positive Borel measure $\mu(d x, d y)$ such that

$$
\int_{X \times X} f(x) \mu(d x, d y)=\int_{X \times X} f(y) \mu(d x, d y)=\int_{X} f(x) d x,
$$

To any volume preserving mapping $h$ in $S$, one can associate a unique doubly stochastic probability measure $\mu_{h}$ defined by

$$
\begin{gathered}
\mu_{h}(d x, d y)=\delta(y-h(x)) d x \\
\text { i.e., } \int_{X \times X} f(x, y) \mu_{h}(d x, d y)=\int_{X} f(x, h(x)) d x \text { for } f \text { in } C(X \times X) .
\end{gathered}
$$

Indeed, if $h$ belongs to $S$, then, for each $f$ in $C(X)$,

$$
\begin{gathered}
\int_{X \times X} f(x) \mu_{h}(d x, d y)=\int_{X} f(x) d x \\
\int_{X \times X} f(y) \mu_{h}(d x, d y)=\int_{X} f(h(x)) d x=\int_{X} f(x) d x \\
\text { (since } h \text { is volume preserving). }
\end{gathered}
$$

By (1.27), $S$ can be identified as a subset of $P$, the set of all doubly stochastic probability measures on $X \times X$. In the same way that $S$ was conjectured to be the strong $L^{2}$ closure of $G$, we believe that $P$ is the weak-* closure of $S$. Once again, since such a result is probably available somewhere in the literature 
and not strictly necessary for our discussion, there will be not attempt to prove it here. It is now tempting to solve the shortest path problem in the class of time parameterized families of doubly stochastic probability measures on $X \times X$ :

$$
t \in[0, T] \rightarrow \mu(t ; d x, d y) \in \operatorname{Prob}(X \times X) .
$$

$\mu(t ; d x, d y)$ can be interpreted as the probability for a particle to go from $x$ at time 0 to $y$ at time $t$. It is now easy to translate the initial and final conditions (1.21), as well as the incompressibility condition (1.22), in terms of $\mu$ :

$$
\mu(0 ; d x, d y)=\delta(y-x) d x, \quad \mu(T ; d x, d y)=\delta(y-h(x)) d x,
$$

$$
\int_{X \times X} f(y) \mu(t ; d x, d y)=\int_{X} f(x) d x, \quad \text { for each } f \text { in } C(X) \text { and } t \text { in }[0, T]
$$

Unfortunately, at this point it becomes extremely difficult to define the Action in terms of $\mu$, and so we give up this approach in this paper. The main problem is the lack of dynamics in the description of a generalized flow as a one parameter family of doubly stochastic probability measures (similar problems have been discussed in the case of hyperbolic systems of conservation laws [5]). Indeed, the Action involves the velocity of the particles, and it is therefore necessary to consider not only the probability that a particle issued from $x$ at time 0 reaches $y$ at time $t$, but also the probability that it goes from $x_{0}$ at time $t_{0}$ to $x_{1}$ at time $t_{1}$ when $t_{1}$ and $t_{0}$ are infinitesimally close, etc. In this paper, a richer concept is used that fully takes into account the dynamics of the particles. To each path $t \in[0, T] \rightarrow z(t) \in X$, one associates the probability that it is followed by some material particle. This defines generalized flows as probability measures on the set $\Omega$ of all possible paths. Obviously, this idea is rather common in both statistical and quantum physics, but, surprisingly, seems ignored in continuum mechanics. It is also closely related to the concept of path integrals [10] as well as the construction of the Wiener integral. The nicest feature of the new framework is the fact that (i) initial, final, and incompressibility conditions are ( $w^{-*}$ continuous) linear constraints on the set $\operatorname{Prob}(\Omega)$, (ii) the Action turns out to be nearly a linear functional. Therefore, we obtain what we could call (following the terminology used for the Monge-Kantorovich problem [9]) a continuous linear programming problem, for which it is rather easy to prove the existence of an optimal generalized flow. Then it is not difficult to check that classical solutions to the Euler equations cannot be missed in our framework. In any case, under some natural restrictions on the time scale, the corresponding flow is the unique solution to the generalized shortest path problem. Moreover, there are examples of optimal generalized flows that are not deterministic and can be explicitly computed. Finally, one can formally derive from any optimal generalized flow a corresponding measure-valued solution to the Euler equations in the sense of DiPerna and Majda. 
The remainder of the paper is organized as follows:

2. A probabilistic concept of generalized flows

3. The generalized shortest path problem

4. A complete existence result in the case of the $d$-dimensional torus

5. Classical solutions and generalized solutions

6. Explicit examples of nondeterministic generalized solutions to the Euler equations

Appendix. A formal link with the measure-valued solutions in the sense of DiPerna and Majda

\section{A PROBABILISTIC CONCEPT OF GENERALIZED FLOWS}

Here $X$ is a compact set in $\mathbf{R}^{d}$ and $d x$ a probability measure on $X$ ( $X$ can be a manifold and $d x$ can be different from the Lebesgue measure). $T>0$ is a fixed time. Sometimes $Q$ will denote the set $[0, T] \times X$ and $\tau$ a generic finite subset $\left\{t_{1}, \ldots, t_{n}\right\}$ of $[0, T]$.

The product space $\Omega=X^{[0, T]}$, which is the set of all paths $z: t \in[0, T] \rightarrow$ $z(t) \in X$, is compact for the product topology. A function $F$ defined on $\Omega$ can be viewed as a path functional and, therefore, the notation $F[t \rightarrow z(t)]$ will sometimes be used instead of $F(z)$. Given a finite subset $\tau=\left\{t_{1}, \ldots, t_{n}\right\}$ of $[0, T]$ and a continuous function $f$ on $X^{n}, F(z)=f\left(z\left(t_{1}\right), \ldots, z\left(t_{n}\right)\right)$ defines a function on $\Omega$ that is always continuous for the product topology [10]. The function(al)s of this kind will be called continuous functionals of finite type. By Stone-Weierstrass' theorem, the set $C_{\text {fin }}(\Omega)$ of all such functionals is a dense subspace of the space $C(\Omega)$ of all continuous functionals on $\Omega . C(\Omega)$ is a Banach space for the sup-norm, and the dual space $C(\Omega)^{\prime}$ is exactly the set of all positive Borel (Radon) measures on $\Omega$. Our first result is elementary but essential for our purposes.

Proposition 2.1. Let $g$ be an incompressible flow on $X$ that reaches a final configuration $h \in S$ at time $T$ (in the sense of (1.21) and (1.22)). Then there exists a unique probability measure $q$ on $\Omega$ defined by

$$
\int_{\Omega} F(z) q(d z)=\int_{X} F[t \rightarrow g(t, x)] d x, \quad \text { for each } F \text { in } C_{\mathrm{fin}}(\Omega) .
$$

$q$ satisfies the following properties:

$$
\int_{\Omega} f(z(0), z(T)) q(d z)=\int_{X \times X} f(x, y) \eta(d x, d y), \quad \text { for each } f \text { in } C(X \times X) .
$$


where $\eta(d x, d y)=\delta(y-h(x)) d x$ defines a doubly stochastic probability measure on $X \times X$, i.e.,

$$
\begin{aligned}
\int_{X \times X} f(x) \eta(d x, d y)=\int_{X \times X} f(y) \eta(d x, d y)= & \int_{X} f(x) d x \\
& \text { for each } f \text { in } C(X) .
\end{aligned}
$$

Proof. If $F$ belongs to $C_{\text {fin }}(\Omega)$, it can be written as $F(z)=f\left(z\left(t_{1}\right), \ldots, z\left(t_{n}\right)\right)$ for some finite set $\left\{t_{1}, \ldots, t_{n}\right\}$ and some continuous function $f$ on $X^{n}$. Thus, formula (2.1), which can be rewritten as

$$
\int_{\Omega} f\left(z\left(t_{1}\right), \ldots, z\left(t_{n}\right)\right) q(d z)=\int_{X} f\left(g\left(t_{1}, x\right), \ldots, g\left(t_{n}, x\right)\right) d x
$$

defines a unique positive linear functional on $C_{\mathrm{fin}}(\Omega)$ and, therefore, by density, a unique positive Borel measure $q$ on $\Omega$. Since $d x$ is a probability measure, $q$ is also a probability measure. Property (2.2) is a straightforward consequence of the incompressibility condition (1.22), and (2.3) immediately follows from conditions (1.21) and definition (2.5). Since $h$ is a volume preserving mapping, the corresponding measure $\eta$ is necessarily a doubly stochastic probability measure, as has been seen in $\S 1$. Proposition 2.1 suggests the following.

Definition 2.2. Any probability measure $q$ on $\Omega$ is called a generalized flow. If (2.2) holds, we say that $q$ is incompressible. Any doubly stochastic probability measure $\eta$ on $X \times X$ is called a (generalized) final configuration. If $q$ satisfies (2.3), we say that $q$ reaches the final configuration $\eta$ at time $T$.

We conjecture that generalized flows and final configurations can be approximated (in the weak-* sense) by classical flows (at least when $X$ is a nice $d$ dimensional domain, with $d \geq 2$ ). Since this result is not, strictly speaking, necessary to our discussion, no proof will be provided here.

As an obvious consequence of Proposition 2.1 and Definition 2.2, we finally get

Proposition 2.3. For the weak ${ }_{-}^{*}$ star topology on $C(\Omega)^{\prime}$, the set of all generalized incompressible flows is compact. The same is true for the set of all generalized flows that reach a given final configuration at a given time $T$, and for the set of all such flows that are incompressible.

\section{THE GENERALIZED SHORTEST PATH PROBLEM}

In order to keep notations as simple as possible, integrals with respect to $t$ (resp. $x, x$ and $y$, and $z$ ) are implicitly performed over $[0, T]$ (resp. $X$, $X \times X$, and $\Omega$ ), the initial density $\rho_{0}$ is simply denoted by $\rho$.

In the previous section, it was established that we can associate to any classical flow $g$ a unique generalized flow $q$ defined as a probability measure on $\Omega$ by (2.1). A formal substitution into (2.1) of $F(z)=\int\left\|z^{\prime}(t)\right\|^{2} d t$ leads to

$$
\iint\left\|z^{\prime}(t)\right\|^{2} d t q(d z)=\iint\left\|\partial_{t} g(t, x)\right\|^{2} d t d x .
$$


In the same way, by substituting for $F$ into (2.1)

$$
a(z)=\rho(z(0))\left\{\frac{1}{2} \int\left\|z^{\prime}(t)\right\|^{2} d t-\int U(t, z(t)) d t\right\},
$$

we get the formal identity

$$
\begin{aligned}
\int \rho(z(0)) & \left\{\frac{1}{2} \int\left\|z^{\prime}(t)\right\|^{2} d t-\int U(t, z(t)) d t\right\} q(d z) \\
= & \iint \rho(x)\left\{\frac{1}{2}\left\|\partial_{t} g(t, x)\right\|^{2}-U(t, g(t, x))\right\} d t d x .
\end{aligned}
$$

These formulae provide formal but simple definitions of the kinetic energy and the Action as linear functionals on the set of all generalized flows. The goal of this section is to get rigorous definitions and, then, to show that the shortest path problem, set in the class of all generalized incompressible flows, always has a solution provided that the final configuration can be reached by at least one generalized incompressible flow having a finite kinetic energy. To state these results, we need

Assumption 3.1. $\rho$ is a lower semicontinuous mapping from $X$ into $[0,+\infty[$ and belongs to $L^{r}(X)$ for some $r, 1 \leq r \leq+\infty ; U$ is in $L^{s}([0, T] \times X)$ if $r>1$, and in $C([0, T] \times X)$ if $r=1$, where $1 / r+1 / s=1$.

Then we have one of our main results.

Theorem 3.2. For any generalized incompressible flow $q$, the generalized kinetic energy

$$
E(q)=\int \rho(z(0)) \int \frac{1}{2}\left\|z^{\prime}(t)\right\|^{2} d t(d z) \text { is well defined in }[0,+\infty] .
$$

If $E(q)$ is finite, then one can define the generalized Action

$$
A(q)=\int \rho(z(0))\left\{\int \frac{1}{2}\left\|z^{\prime}(t)\right\|^{2} d t-\int U(t, z(t)) d t\right\} q(d z) .
$$

For any generalized final configuration $\eta$, if there is one generalized incompressible flow that can reach $\eta$ at time $T$ with a finite kinetic energy, then there is such a flow that minimizes the Action.

The proof is based on several technical results.

Proposition 3.3. For any $z$ in $\Omega$, let us define

$$
\begin{aligned}
e(z) & =0 \quad \text { if } \rho(z(0))=0, \\
& =\rho(z(0)) \int \frac{1}{2}\left\|z^{\prime}(t)\right\|^{2} d t \quad \text { if } z \text { is in the Sobolev space } H^{1}[0, T] \\
& =+\infty \text { otherwise. }
\end{aligned}
$$

Then $e$ is a lower semicontinuous mapping from $\Omega$ into $[0,+\infty]$, and the integral

$$
E(q)=\int e(z) q(d z)
$$


defines a (weak-*) lower semicontinuous mapping from the set of all probability measures on $\Omega, \operatorname{Prob}(\Omega)$, into $[0,+\infty]$. Moreover,

$$
E(q)=\sup \left\{\int e_{\tau}(z) q(d z) ; \tau \text { finite subset of }[0, T]\right\},
$$

where

$$
\begin{aligned}
& e_{\tau}(z)=\rho(z(0)) \sum \frac{1}{2}\left\|z\left(t_{k}\right)-z\left(t_{k-1}\right)\right\|^{2}\left(t_{k}-t_{k-1}\right)^{-1}, \\
& \text { for } \tau=\left\{t_{1}, \ldots, t_{n}\right\}, 0 \leq t_{1} \leq \cdots \leq t_{n} \leq T,
\end{aligned}
$$

defines a lower semicontinuous mapping from $\Omega$ into $[0,+\infty[$.

Proposition 3.4. For any generalized incompressible flow $q$ such that $E(q)$ is finite,

$$
V(z)=\rho(z(0)) \int U(t, z(t)) d t
$$

is well defined as a q-integrable function on $\Omega$ and

$$
\begin{gathered}
\int|V(z)| q(d z) \leq\|\rho\|_{L^{r}}\|U\|_{L^{s}} . \\
\text { If } \rho=\mathrm{cst}=1, \text { then } \int V(z) q(d z)=\iint U(t, x) d t d x .
\end{gathered}
$$

Proposition 3.5. If $\left(q_{m}\right)$ is a sequence of generalized incompressible flows, with uniformly bounded kinetic energy, that converges toward $q$ in the weak-* sense, then

$$
E(q) \leq \liminf E\left(q_{m}\right) \text { and } \int V(z) q(d z)-\lim \int V(z) q_{m}(d z) .
$$

Proof of Proposition 3.3. According to classical results [3] on the integration of lower semicontinuous (1.s.c.) functions valued in $[0,+\infty]$, it is enough to prove that (i) the family $\left(e_{\tau}\right)$, where the $\tau=\left\{t_{1}, \ldots, t_{n}\right\}, 0 \leq t_{1}<\cdots<t_{n} \leq T$, are arbitrary finite subsets of $[0, T]$, is a filtering increasing family of positive 1.s.c. functions defined on $\Omega$, (ii) $e$ can be written as the supremum of the $e_{\tau}$.

(i) $\rho$ is a positive 1.s.c. mapping from $X$ into $[0,+\infty[$ and, thus, $z \rightarrow$ $\rho(z(0))$ is also positive and 1.s.c. on $\Omega$ for the product topology; $z \rightarrow$ $\sum\left\|z\left(t_{k}\right)-z\left(t_{k-1}\right)\right\|^{2}\left(t_{k}-t_{k-1}\right)^{-1}$ is a positive functional of finite type, continuous on $\Omega$. Therefore, as a product of a l.s.c. function by a continuous function, both valued in $\left[0,+\infty\left[\right.\right.$, each $e_{\tau}$ is also 1.s.c. and valued in $\left[0,+\infty\left[\right.\right.$. The fact that $\left(e_{\tau}\right)$ (which is not a countable family!) is filtering increasing follows from the Cauchy-Schwarz inequality. Indeed, for any sequence $0 \leq t_{1}<\cdots<t_{n} \leq T$, we have

$$
\left\|z\left(t_{n}\right)-z\left(t_{1}\right)\right\|^{2}\left(t_{n}-t_{1}\right)^{-1} \leq \sum_{k=2, n}\left\|z\left(t_{k}\right)-z\left(t_{k-1}\right)\right\|^{2}\left(t_{k}-t_{k-1}\right)^{-1} .
$$

Therefore, given two finite sets $\tau_{1}, \tau_{2}$ in $[0, T]$, by setting $\tau_{3}=\tau_{1} \cup \tau_{2}$, one easily deduces $e_{\tau_{3}} \geq \max \left(e_{\tau_{1}}, e_{\tau_{2}}\right)$. This precisely means that $\left(e_{\tau}\right)$ is filtering increasing. 
(i) Let us now prove that $e=e^{\#}$, where $e^{\#}=\sup e_{\tau}$, in two steps:

(a) if $z$ belongs to $H^{1}[0, T]$, then $e^{\#}(z) \leq e(z)<+\infty$;

(b) if $e^{\#}(z)<+\infty$, then either $\rho(z(0))=0$ and $e^{\#}(z)=e(z)$ or $z$ belongs to $H^{1}[0, T]$ and $e(z) \leq e^{\#}(z)$.

The first statement is another trivial consequence of the Cauchy-Schwarz inequality, since, for any $z$ in $H^{1}[0, T]$ and any set $\tau=\left\{t_{1}, \ldots, t_{n}\right\}, 0 \leq$ $t_{1}<\cdots<t_{n} \leq T$, we have

$$
\sum_{k=2, n}\left\|z\left(t_{k}\right)-z\left(t_{k-1}\right)\right\|^{2}\left(t_{k}-t_{k-1}\right)^{-1} \leq \int\left\|z^{\prime}(t)\right\|^{2} d t,
$$

and, thus, $e_{\tau}(z) \leq e(z)$.

Let us now prove the second statement. Let $z$ be a path such that $e^{\#}(z)<$ $+\infty$. If $\rho(z(0))=0$, then both $e(z)$ and $e^{\#}(z)$ are equal to zero. Let us consider the case when $\rho(z(0))>0$. For each pair $t_{1}<t_{2}$, we get for $\tau=$ $\left\{t_{1}, t_{2}\right\}$

$$
e_{\tau}(z)=\rho(z(0))\left\|z\left(t_{2}\right)-z\left(t_{1}\right)\right\|^{2}\left(t_{2}-t_{1}\right)^{-1} \leq e^{\#}(z)<+\infty .
$$

Thus, $z$ is necessarily Hölder continuous from $[0, T]$ into $X$, and it makes sense to consider its derivative $z^{\prime}$ in the sense of distributions. For any $C^{\infty}$ compactly supported mapping $\zeta$ from $[0, T]$ into $\mathbf{R}^{d},\left\langle z^{\prime}, \zeta\right\rangle=-\int \zeta^{\prime}(t)$. $z(t) d t$. Since $\zeta, \zeta^{\prime}$, and $z$ are continuous, for any $\varepsilon>0$, we can find a finite set $\tau=\left\{t_{1}, \ldots, t_{n}\right\}, 0=t_{1}<\cdots<t_{n}=T$, such that

$$
\begin{aligned}
& \left|-\left\langle z^{\prime}, \zeta\right\rangle-\sum_{k=1, n-1}\left(\zeta\left(t_{k+1}\right)-\zeta\left(t_{k}\right)\right) z\left(t_{k}\right)\right| \leq \varepsilon, \\
& \left|\sum_{k=2, n}\left\|\zeta\left(t_{k}\right)\right\|^{2}\left(t_{k}-t_{k-1}\right)-\int\|\zeta(t)\|^{2} d t\right| \leq \varepsilon .
\end{aligned}
$$

Since $\zeta$ vanishes at 0 and $T$, we get

$$
\begin{aligned}
\sum_{k=1, n-1}\left(\zeta\left(t_{k+1}\right)-\zeta\left(t_{k}\right)\right) z\left(t_{k}\right)= & -\sum_{k=2, n}\left(z\left(t_{k}\right)-z\left(t_{k-1}\right)\right) \zeta\left(t_{k}\right) \\
\leq & \left(\sum_{k=2, n}\left\|z\left(t_{k}\right)-z\left(t_{k-1}\right)\right\|^{2}\left(t_{k}-t_{k-1}\right)^{-1}\right)^{1 / 2} \\
& \cdot\left(\sum_{k=2, n}\left\|\zeta\left(t_{k}\right)\right\|^{2}\left(t_{k}-t_{k-1}\right)\right)^{1 / 2} \\
\leq & \left(\rho(z(0))^{-1} e^{\#}(z)\right)^{1 / 2}\left(\int\|\zeta(t)\|^{2} d t+\varepsilon\right)^{1 / 2}
\end{aligned}
$$


Thus,

$$
\left|\left\langle z^{\prime}, \zeta\right\rangle\right| \leq \varepsilon+\left(\rho(z(0))^{-1} e^{\#}(z)\right)^{1 / 2}\left(\int\|\zeta(t)\|^{2} d t+\varepsilon\right)^{1 / 2}
$$

Since $\varepsilon$ is arbitrary, it follows that $z$ belongs to $H^{1}[0, T]$ and $\int\left\|z^{\prime}(t)\right\|^{2} d t \leq$ $\rho(z(0))^{-1} e^{\#}(z)$. Thus, $e(z) \leq e^{\#}(z)$, which proves statement (b) and, therefore, achieves the proof of Proposition 3.3.

Proof of Propositions 3.4 and 3.5. Since $\rho$ is a positive integrable 1.s.c. function on $X$, for each integer $n>0$, there exists a continuous approximation $\rho_{n}$ such that $0 \leq \rho_{n} \leq \rho$ and $\left\|\rho_{n}-\rho\right\|_{L^{1}} \leq \frac{1}{n}$. Since $U$ is in $L^{s}([0, T] \times X)$ if $r>1$, and in $C([0, T] \times X)$ if $r=1$, where $\frac{1}{r}+\frac{1}{s}=1$, there is, for each integer $m>0$, a Lipschitz continuous approximation $U_{m}$ such that $\left\|U_{m}-U\right\|_{L^{s}} \leq \frac{1}{m}$.

To show that $V(z)=\rho(z(0)) \int U(t, z(t)) d t$ can be well defined as a $q$ integrable function for any generalized flow $q$ having a finite kinetic energy $E(q)=\int^{*} e(z) q(d z)<+\infty$, let us introduce, for any integers $n, m, k>0$, the following approximations to $V$ (where $T=1$ is set for simplicity):

$$
\begin{aligned}
& V_{n m k}(z)=\rho_{n}(z(0)) k^{-1} \sum_{j=1, k} U_{m}\left(\frac{j}{k}, z\left(\frac{j}{k}\right)\right), \\
& V_{n m}(z)=0 \text { if } \rho_{n}(z(0))=0, \\
& =\rho_{n}(z(0)) \int U_{m}(t, z(t)) d t \text { if } z \text { belongs to } H^{1} .
\end{aligned}
$$

Notice that $V_{n m}$ is defined $q$-almost everywhere, since $E(q)$ is finite, which implies $q$-almost surely either $\rho(z(0))=0$ (and, therefore, $\rho_{n}(z(0))=0$ ) or $z \in H^{1}$. Also notice that, in the special case when $\rho$ is identically equal to 1 , we can take $\rho_{n}$ identically equal to 1 , and, then, since $q$ is assumed to be incompressible

$$
\int V_{n m k}(z) q(d z)=k^{-1} \sum_{j=1, k} \int U_{m}\left(\frac{j}{k}, x\right) d x \quad(\text { if } \rho=1) .
$$

By integrating in $z$ over $\Omega$, we get for any $n, n^{\prime}, m, k$,

$$
\begin{aligned}
\int\left|V_{n m k}(z)-V_{n^{\prime} m k}(z)\right| q(d z) & \leq \sup \left|U_{m}\right| \int\left|\rho_{n}(z(0))-\rho_{n^{\prime}}(z(0))\right| q(d z) \\
& =\sup \left|U_{m}\right|\left\|\rho_{n}-\rho_{n^{\prime}}\right\|_{L^{\prime}}
\end{aligned}
$$


(since $q$ is incompressible). By using Hölder inequalities (twice), we also get for any $n, m, m^{\prime}, k$,

$$
\begin{aligned}
& \int\left|V_{n m k}(z)-V_{n m^{\prime} k}(z)\right| q(d z) \\
& \leq\left(\int \rho_{n}(z(0))^{r} q(d z)\right)^{1 / r} \\
& \cdot\left(k^{-1} \sum_{j=1, k} \int\left|U_{m}\left(\frac{j}{k}, z\left(\frac{\dot{k}}{k}\right)\right)-U_{m^{\prime}}\left(\frac{\dot{k}}{k}, z\left(\frac{j}{k}\right)\right)\right|^{s} q(d z)\right)^{1 / s} \\
&=\left(\int \rho_{n}(x)^{r} d x\right)^{1 / r} \\
& \cdot\left(k^{-1} \sum_{j=1, k} \int\left|U_{m}\left(\frac{j}{k}, x\right)-U_{m^{\prime}}\left(\frac{j}{k}, x\right)\right|^{s} d x\right)^{1 / s}
\end{aligned}
$$

(since $q$ is incompressible). It follows that

$$
\left\|V_{n m k}-V_{n m^{\prime} k}\right\|_{L^{1}} \leq\|\rho\|_{L^{r}}\left(\left\|U_{m}-U_{m^{\prime}}\right\|_{L^{s}}+\delta(k)\left(c_{m}+c_{m^{\prime}}\right)\right),
$$

where $c_{m}$ depends only on $U_{m}$ and $\delta(k)$ tends to 0 when $k$ tends to $+\infty$. In the same way, we obtain

$$
\left\|V_{n m k}\right\|_{L^{1}} \leq\|\rho\|_{L^{r}}\left(\left\|U_{m}\right\|_{L^{s}}+\delta(k) c_{m}\right) .
$$

In the special case $\rho=1$, we also get

$$
\left|\int V_{n m k}(z) q(d z)-\iint U_{m}(t, x) d t d x\right| \leq k^{-1} c_{m} .
$$

Let us now consider a path $z$ such that $\rho(z(0))>0$. It belongs to $H^{1}[0, T]$ $q$-almost surely and, therefore,

$$
\begin{aligned}
\left|V_{n m k}(z)-V_{n m}(z)\right| & \leq \rho_{n}(z(0)) k^{-1} c_{m} \int\left(1+\left|z^{\prime}(t)\right|\right) d t \\
& \leq 2 \rho_{n}(z(0)) k^{-1} c_{m}\left(\int\left(1+\left|z^{\prime}(t)\right|^{2}\right) d t\right)^{1 / 2} .
\end{aligned}
$$

If $\rho(z(0))=0$, then $\rho_{n}(z(0))=0$, and the right-hand side is equal to zero. Thus, after integrating with respect to $z$ over $\Omega$, we get (by using the Schwarz inequality)

$$
\begin{aligned}
& \int\left|V_{n m k}(z)-V_{n m}(z)\right| q(d z) \\
& \quad \leq 2 k^{-1} c_{m}\left(\int \rho_{n}(z(0)) q(d z) \int \rho_{n}(z(0)) \int\left(1+\left|z^{\prime}(t)\right|^{2}\right) d t q(d z)\right)^{1 / 2} .
\end{aligned}
$$

Since $q$ is incompressible, we have

$$
\int \rho_{n}(z(0)) q(d z)=\int \rho_{n}(x) d x \leq \int \rho(x) d x,
$$


and it follows that

$$
\left\|V_{n m k}-V_{n m}\right\|_{L^{1}} \leq \frac{1}{k} c c_{m} \text { (where } c \text { depends only on } E(q) \text { and }\|\rho\|_{L^{1}} \text { ). }
$$

These estimates show that, for any integer $i>0$, we can choose $m=m_{i}$, then $n=n_{i}, k=k_{i}$, in such a way that $W_{i}=V_{n_{i} m_{i} k_{i}}$ satisfies

$$
\begin{gathered}
\left\|V_{n_{i} m_{i}}-W_{i}\right\|_{L^{1}} \leq \frac{1}{i}, \\
\left\|W_{i^{\prime}}-W_{i}\right\|_{L^{1}} \leq \frac{1}{i}+\frac{1}{i^{\prime}}, \\
\left\|W_{i}\right\|_{L^{1}} \leq\|\rho\|_{L^{r}}\left(\|U\|_{L^{s}}+\frac{1}{i}\right),
\end{gathered}
$$

and

$$
\left|\int W_{i}(z) q(d z)-\iint U(t, x) d t d x\right| \leq \frac{1}{i} \quad \text { if } \rho=1 .
$$

It follows that $\left(W_{i}\right)$ is a Cauchy sequence in $L^{1}$ and has a unique limit $V$ that can be formally defined by (3.10),

$$
V(z)=\rho(z(0)) \int U(t, z(t)) d t .
$$

Moreover,

$$
\|V\|_{L^{\prime}} \leq\|\rho\|_{L^{\prime}}\|U\|_{L^{\prime}}
$$

and

$$
\int V(z) q(d z)=\iint U(t, x) d t d x \quad \text { if } \rho=1 .
$$

This achieves the proof of Proposition 3.4.

To prove Proposition 3.5, let us consider a sequence $\left(q_{m}\right)$ of generalized incompressible flows that weak-* converges toward $q$ and satisfies $\sup E\left(q_{m}\right)<$ $+\infty$. Necessarily, $q$ belongs to the set of all generalized incompressible flows since this set is weak-* closed (Proposition 2.3). Moreover, the generalized kinetic energy $E$ is weak-* 1 .s.c. Therefore, $E(q) \leq \liminf E\left(q_{m}\right)<+\infty$, which proves the first part of Proposition 3.5. To prove the remainder, let us first remark that all the estimates that have been previously obtained for the Cauchy sequence $\left(W_{i}\right)$ are uniform in $q$, as long as $E(q)$ stays uniformly bounded. It follows that the $L^{1}\left(\Omega, q_{m}\right)$ norm of $V-W_{i}$ uniformly goes to 0 when $i$ tends to $+\infty$. Since each $W_{i}$ is a continuous functional (of finite type) and $q_{m}$ converges toward $q$ in the weak-* sense, we get, for a fixed $i$,

$$
\int W_{i}(z) q(d z)=\lim \int W_{i}(z) q_{m}(d z) .
$$

Then the fact that $V$ is uniformly (with respect to $m$ ) approximated by $W_{i}$ shows that $V$ is $q$-integrable and

$$
\int V(z) q(d z)=\lim \int V(z) q_{m}(d z) .
$$


This completes the proof of Proposition 3.5.

Proof of Theorem 3.2. Let us call $P_{\eta}$ the set of all generalized incompressible flows $q$ that reach $\eta$ at time $T$ with a finite kinetic energy $E(q)<+\infty$. By Proposition 3.4, for each $q$ in $P_{\eta}, V(z)=\rho(z(0)) \int U(t, z(t)) d t$ defines a $q$-integrable function and, by (3.11), $\int|V(z)| q(d z)$ is uniformly bounded by a constant $C$ that depends only on $\rho$ and $U$. Therefore, we get for each $q$ in $P_{\eta}$

$$
-\infty<-C \leq A(q)=\int(V(z)+e(z)) q(d z) \leq E(q)+C<+\infty .
$$

Thus, there certainly exists a minimizing sequence $\left(q_{m}\right)$ in $P_{\eta}$ such that

$$
\lim A\left(q_{m}\right)=A_{\mathrm{opt}}=\operatorname{Inf}\left\{A(q), q \text { in } P_{\eta}\right\} \quad \text { and } \quad C^{\prime}=\sup A\left(q_{m}\right)<+\infty
$$

By Proposition 2.3, it is not a restriction to suppose that $\left(q_{m}\right)$ converges toward a generalized incompressible flow $q$ that reaches $\eta$ at time $T$. Moreover, since $\sup E\left(q_{m}\right) \leq C+C^{\prime}<+\infty$, Proposition 3.5 can be used. Thus, $E(q) \leq$ $\lim \inf E\left(q_{m}\right)$ and $\int V(z) q(d z)=\lim V(z) q_{m}(d z)$. It follows that $q$ has a finite kinetic energy and $A(q) \leq \lim A\left(q_{m}\right)=A_{\text {opt }}$, which shows that $q$ is optimal and completes the proof.

Added in Proof. In the proof of Theorem 3.2, it is implicitly stated that a bounded sequence in the dual space $C(\Omega)^{\prime}$ always has a convergent subsequence. This is not correct since, a priori, $C(\Omega)$ is not separable. Such a sequence always has a cluster point, no more. As a matter of fact, nets should be used instead of sequences. This does not affect the proof in any essential way, but Proposition 3.5 should be modified to take nets into account.

\section{A COMPLETE EXISTENCE RESULT \\ IN THE CASE OF THE $d$-DIMENSIONAL TORUS}

By Theorem 3.2, we know that, for a given $T>0$ and a given final configuration $\eta$, there exists an optimal generalized incompressible flow that minimizes the Action, provided that $\eta$ can be reached at time $T$ by at least one generalized incompressible flow with finite kinetic energy. Thus, our main concern is now to exhibit such a flow. A complete answer can be obtained in the case of the $d$-dimensional torus $X=\mathbf{R}^{d} / \mathbf{Z}^{d}$ (that is the $d$-dimensional unit cube with periodic boundary conditions) or, slightly more generally, in the case when $X$ 
and $d x$ satisfy

Property 4.1. There exist a constant $C$ and a measurable mapping $(t, x, y) \rightarrow$ $\gamma(t, x, y)$ from $[0,1] \times X \times X$ into $X$ such that

$$
\begin{aligned}
\gamma(0, x, y) & =x, \quad \gamma(1, x, y)=y ; \\
\int\left\|\partial_{t} \gamma(t, x, y)\right\|^{2} d t \leq C, & \text { for almost every } x, y \text { in } X ; \\
\iint f(\gamma(t, x, y)) d x d y & =\int f(x) d x, \\
\text { for any } f & \text { in } C(X) \text { and any } t \text { in }[0,1] .
\end{aligned}
$$

This property is satisfied by $X=\mathbf{R}^{d} / \mathbf{Z}^{d}$ and $d x=$ Lebesgue measure, once we define $t \rightarrow \gamma(t, x, y)$ to be the geodesical path between $x$ and $y$, which is uniquely defined for almost every pair of points on the torus. Indeed, (4.1) and (4.2) are obvious and, from the straightforward translation invariance property

$$
\gamma(t, x, y)=x+\gamma(t, 0, y-x),
$$

we easily deduce for any $f$ in $C(X)$

$$
\begin{aligned}
\iint f(\gamma(t, x, y)) d x d y & =\iint f(x+\gamma(t, 0, y-x)) d x d y \\
& =\iint f\left(x+\gamma\left(t, 0, y^{\prime}\right)\right) d x d y^{\prime} \\
& =\iint f(x) d x d y^{\prime}=\int f(x) d x
\end{aligned}
$$

Thanks to Property 4.1, we can prove our main result.

Theorem 4.2. Assume that $X=\mathbf{R}^{d} / \mathbf{Z}^{d}$ (or Property 4.1 holds); $\rho$ is a lower semicontinuous mapping from $X$ into $\left[0,+\infty\left[\right.\right.$ and belongs to $L^{r}(X)$ for some $r, 1 \leq r \leq \infty ; U$ is in $L^{s}([0, T] \times X)$ if $r>1$, and in $C([0, T] \times X)$ if $r=1$, where $\frac{1}{r}+\frac{1}{s}=1$. Then, for any final configuration $\eta$ (i.e., any doubly stochastic probability measure on $X \times X)$ and any final time $T>0$, there exists a generalized incompressible flow that reaches $\eta$ at time $T$ with a finite kinetic energy and minimizes the Action

$$
A(q)=\int \rho(z(0))\left\{\int \frac{1}{2}\left\|z^{\prime}(t)\right\|^{2} d t-\int U(t, z(t)) d t\right\} q(d z) .
$$

This result is a straightforward corollary of Theorem 3.2 and

Proposition 4.3. If $X=\mathbf{R}^{d} / \mathbf{Z}^{d}$, then for any final configuration $\eta$ and any final time $T>0$, there exists a generalized incompressible flow $q$ that reaches $\eta$ at time $T$ with a finite kinetic energy

$$
E(q)=\int \rho(z(0)) \int \frac{1}{2}\left\|z^{\prime}(t)\right\|^{2} d t q(d z) \leq \frac{2 C}{T} \int \rho(x) d x,
$$

where $C$ is the constant considered in Property 4.1. 
Proof of Proposition 4.3. Let us explicitly define $q$ by

$$
\int F(z) q(d z)=\iiint F\left[t \rightarrow G\left(t, x, x^{\prime}, y\right)\right] \eta(d x, d y) d x^{\prime},
$$

for each $F$ in $C_{\text {fin }}(\Omega)$,

where

$$
\begin{aligned}
G\left(t, x, x^{\prime}, y\right) & =\gamma\left(\frac{2 t}{T}, x, x^{\prime}\right), \quad \text { if } 0 \leq t \leq \frac{T}{2} \\
& =\gamma\left(2-\frac{2 t}{T}, x^{\prime}, y\right), \quad \text { if } \frac{T}{2} \leq t \leq T .
\end{aligned}
$$

This intuitively means that a particle issued from $x$ at time 0 can reach at time $\frac{T}{2}$ any point $x^{\prime}$ in $X$ with the same uniform probability and then reaches $y$ at time $T$ according to the probability law $\eta(d x, d y)$. During the intermediate times, $0 \leq t \leq \frac{T}{2}$ and $\frac{T}{2} \leq t \leq T$, each particle follows a geodesic on the torus. To prove Proposition 4.3, it is sufficient to check that (i) $q$ is a generalized incompressible flow, (ii) $q$ reaches $\eta$ at time $T$, and (iii) $q$ has a finite kinetic energy.

(i) $G$ is a measurable mapping from $[0,1] \times X \times X$ into $X$, and (4.5) defines a generalized flow on $X$. To check that $q$ is incompressible, let us fix $t$ in $[0, T]$ and $f$ in $C(X)$. For $0 \leq t \leq \frac{T}{2}$, we have

$$
\begin{aligned}
\int f(z(t)) q(d z) & =\iiint f\left(\gamma\left(\frac{2 t}{T}, x, x^{\prime}\right)\right) \eta(d x, d y) d x^{\prime} \\
& =\iint f\left(\gamma\left(\frac{2 t}{T}, x, x^{\prime}\right)\right) d x d x^{\prime} \quad \text { (by definitions (4.5) and (4.6)) } \eta \text { is doubly stochastic) } \\
& =\int f(x) d x \quad \text { (by property (4.3)). }
\end{aligned}
$$

We get the same result when $\frac{T}{2} \leq t \leq T$, for the same reasons.

(ii) For any $f$ in $C(X \times X)$, we have

$$
\begin{aligned}
\int f(z(0), z(T)) q(d z) & =\iiint f\left(G\left(0, x, x^{\prime}, y\right), G\left(T, x, x^{\prime}, y\right)\right) \eta(d x, d y) d x^{\prime} \\
& =\iiint f(x, y) \eta(d x, d y) d x^{\prime} \quad \text { (by definition (4.5)) } \\
& =\iint f(x, y) \eta(d x, d y) .
\end{aligned}
$$

Thus, $q$ reaches $\eta$ at time $T$. 
(iii) By definition of $q$, we immediately get

$$
\begin{gathered}
E(q)=\iiint \rho\left(G\left(0, x, x^{\prime}, y\right)\right)\left[\int \frac{1}{2}\left\|\partial_{t} G\left(t, x, x^{\prime}, y\right)\right\|^{2} d t\right] \eta(d x, d y) d x^{\prime} \\
=\frac{1}{T} \iiint \rho(x)\left[\int\left\|\partial_{t} \gamma\left(\tau, x, x^{\prime}\right)\right\|^{2} d \tau\right. \\
\left.+\int\left\|\partial_{t} \gamma\left(\tau, x^{\prime}, y\right)\right\|^{2} d \tau\right] \eta(d x, d y) d x^{\prime}
\end{gathered}
$$

(by definition (4.6); here the $d \tau$-integrals are performed over the unit interval $[0,1])$ $\leq \frac{2 C}{T} \iiint \rho(x) \eta(d x, d y) d x^{\prime} \quad$ (by definition of $C$ in Property 4.1) $=\frac{2 C}{T} \int \rho(x) d x \quad$ (since $\eta$ is doubly stochastic).

This completes the proof of Proposition 4.3.

\section{Classical solutions AND GeNERAlized SOlutions}

In this section, it is shown that, under a natural restriction on the time scale, any classical solution to the Euler equations satisfies the generalized Least Action Principle and is the unique solution to the corresponding generalized shortest path problem. This is a consequence of the following result.

Theorem 5.1. Let $q$ be a generalized incompressible flow, and let $\eta$ be the final configuration that it reaches at time $T$. Assume that

$$
\rho \text { is bounded away from zero by some constant } \alpha>0 \text {; }
$$

$U$ is Lipschitz continuous on $Q=[0, T] \times X$, and there is a constant $C$ such that

(5.2) $D^{2} U(t, x) \leq C \quad$ (in the sense of distributions and symmetric matrices);

there exists a Lipschitz continuous pressure field $p(t, x)$ on $Q$ such that (5.3)

$$
D^{2} p(t, x) \leq R \text { holds (in the same sense as above) for some real constant } R \text {; }
$$

q-almost surely, a path $z$ in $\Omega$ belongs to the Sobolev space $H^{1}[0, T]$ and satisfies the ordinary differential equation

$\rho(z(0))\left\{z^{\prime \prime}(t)+\operatorname{grad} U(t, z(t))\right\}+\operatorname{grad} p(t, z(t))=0, \quad$ in an appropriate sense;

the kinetic energy $E(q)$ is finite. Then $q$ satisfies the generalized Least Action Principle in the sense that it minimizes the Action

$$
A(q)=\int \rho(z(0))\left\{\frac{1}{2}\left\|z^{\prime}(t)\right\|^{2} d t-\int U(t, z(t)) d t\right\} q(d z)
$$


among all generalized incompressible flows that reach the same final configuration $\eta$ at time $T$, provided that inequality

$$
\left(R \alpha^{-1}+C\right) T^{2} \leq \pi^{2}
$$

holds. If this inequality is strict, then $q$ is the unique minimizer and has the following deterministic property: q-almost surely, two paths $z$ and $z^{\#}$ that satisfy $z(0)=z^{\#}(0)$ and $z(T)=z^{\#}(T)$ are equal. Conversely, there are cases when either $q$ is not the unique minimizer or $q$ is not deterministic and

$$
\left(R \alpha^{-1}+C\right) T^{2}=\pi^{2} .
$$

Before proving Theorem 5.1, let us introduce some notation and prove some technical results. For any path $z$ in $H^{1}[0, T]$, let us define

$$
B(z)=\int\left\{\rho(z(0))\left[\frac{1}{2}\left\|z^{\prime}(t)\right\|^{2}-U(t, z(t))\right]-p(t, z(t))\right\} d t,
$$

and, for any $x$ and $y$ in $X$,

$b(x, y)=\operatorname{Inf}\left\{B(z), z \in \Omega, z\right.$ belongs to $H^{1}[0, T], z(0)=x$, and $\left.z(T)=y\right\}$.

Then we have

Lemma 5.2. Under the same assumptions as in Theorem 5.1, if inequality (5.5) holds, then q-almost every path $z$ satisfies

$$
B(z)=b(z(0), z(T)) .
$$

If the inequality is strict, then, for any path $z^{\#}$ that belongs to $H^{1}[0, T]$,

$$
B(z)=B\left(z^{\#}\right), z(0)=z^{\#}(0), z(T)=z^{\#}(T) \text { imply } z=z^{\#} .
$$

Proof of Lemma 5.2. Let us first remark that, by definitions (5.7) and (5.8) of $B$ and $b$, we obviously have

$$
B(z) \geq b(z(0), z(T)), \quad \text { for any path } z \text { that belongs to } H^{1}[0, T] .
$$

Lemma 5.2 says that the corresponding equality holds $q$-almost everywhere on $\Omega$ as soon as inequality (5.5) is satisfied. Moreover, it says that, $q$-almost surely, there is only one $z$ such that $B(z)=b(z(0), z(T))$ when $z(0)$ and $z(T)$ are prescribed and inequality (5.5) is strict.

Let us pick up an arbitrary path $z$ in $\Omega$. By assumption, $q$-almost surely, $z$ is in $H^{1}[0, T]$ and is a solution to the ordinary differential equation (5.4), which means exactly that the first variation of $B\left(z^{\#}\right)$ (defined by (5.7)) vanishes at $z^{\#}=z$, when $z^{\#}$ is an arbitrary path in $H^{1}[0, T]$ such that $z^{\#}(0)=z(0)$ and $z^{\#}(T)=z(T)$. It does not a priori mean that $B\left(z^{\#}\right)$ is minimal at $z^{\#}=z$. This is why we need inequality (5.5) to make sure that $z$ actually is a minimum point (and not a maximum or a saddle point). The proof is an elementary application of the Poincare inequality. Let us first introduce some notation.

$$
\beta=\rho(z(0)), \quad K=\beta C+R \quad(C \text { and } R \text { are defined in (5.2) and (5.3)) }
$$




$$
\begin{aligned}
\phi(t, x) & =\frac{K}{2}\|x\|^{2}-\beta U(t, x)-p(t, x) \\
& =\beta\left(\frac{1}{2} C\|x\|^{2}-U(t, x)\right)+\frac{1}{2} R\|x\|^{2}-p(t, x) .
\end{aligned}
$$

Since both $p$ and $U$ are Lipschitz continuous on $Q$ and satisfy (5.2) and (5.3), it follows that $\phi(t, x)$ is Lipschitz continuous and convex in $x$. Thus, we get (5.14)

$\phi\left(t, z^{\#}(t)\right) \geq \phi(t, z(t))+w(t) \cdot\left(z^{\#}(t)-z(t)\right), \quad$ for all paths $z^{\#}, z$ in $H^{1}[0, T]$, and any measurable curve $t \in[0, T] \rightarrow w(t) \in \mathbf{R}^{d}$ such that

$$
\begin{aligned}
w(t) & \in \partial \phi(t, z(t)) \quad \text { a.e. on } 0 \leq t \leq T, \\
& \text { where } \partial \phi(t, \cdot) \text { is the subdifferential of } \phi(t, \cdot) .
\end{aligned}
$$

Equation (5.4) can be rewritten more accurately as a multivocal o.d.e.

$$
\beta z^{\prime \prime}(t)+K z(t) \in \partial \phi(t, z(t)), \quad \text { a.e. on } 0 \leq t \leq T,
$$

which means

$$
\int\left\{-\beta z^{\prime}(t) \cdot\left[z^{\# \prime}(t)-z^{\prime}(t)\right]+[K z(t)-w(t)] \cdot\left[z^{\#}(t)-z(t)\right]\right\} d t=0,
$$

for any path $z^{\#}$ in $H^{1}[0, T]$ such that $z^{\#}(0)=z(0), z^{\#}(T)=z(T)$, and some curve $w$ that satisfies (5.15). Because of the convexity of $\phi$, it follows that

$$
\begin{aligned}
\int\{- & \left.\beta z^{\prime}(t) \cdot\left[z^{\# \prime}(t)-z^{\prime}(t)\right]+K z(t) \cdot\left[z^{\#}(t)-z(t)\right]\right\} d t \\
\leq & \int \phi\left(t, z^{\#}(t)-\phi(t, z(t))\right] d t,
\end{aligned}
$$

that is

$$
\begin{aligned}
& \int\left\{\left[\frac{\beta}{2}\left\|z^{\prime}(t)\right\|^{2}-\frac{K}{2}\|z(t)\|^{2}+\phi(t, z(t))\right]\right. \\
& \left.-\left[\frac{\beta}{2}\left\|z^{\# \prime}(t)\right\|^{2}-\frac{K}{2}\left\|z^{\#}(t)\right\|^{2}+\phi\left(t, z^{\#}(t)\right)\right]\right\} d t \\
& \quad \leq \int\left\{-\frac{\beta}{2}\left\|z^{\# \prime}(t)-z^{\prime}(t)\right\|^{2}+\frac{K}{2}\left\|z^{\#}(t)-z(t)\right\|^{2}\right\} d t .
\end{aligned}
$$

The left-hand side is precisely $B(z)-B\left(z^{\#}\right)$ (by definition (5.7), (5.12), and (5.13) of $K, \beta, \phi$, and $B$ ), and the right-hand side can be estimated with the help of Poincaré's inequality

$$
\int\left\|z^{\#}(t)-z(t)\right\|^{2} d t \leq\left(\frac{T}{\pi}\right)^{2} \int\left\|z^{\# \prime}(t)-z^{\prime}(t)\right\|^{2} d t
$$

since $z^{\sharp}(0)-z(0)=z^{\sharp}(T)-z(T)=0$. Thus, we get

$$
B(z)-B\left(z^{\#}\right) \leq \frac{1}{2}\left\{\left(\left(\frac{T}{\pi}\right)^{2} K-\beta\right) \int\left\|z^{\# \prime}(t)-z^{\prime}(t)\right\|^{2} d t .\right.
$$

By definition (5.12) and (5.13) of $K$ and $\beta,\left(\frac{T}{\pi}\right)^{2} K-\beta$ is precisely negative when inequality (5.5) holds. Therefore, $B(z)-B\left(z^{\#}\right) \leq 0$, which proves that 
$z$ minimizes $B$ among all paths $z^{\#}$ in $H^{1}[0, T]$ such that $z^{\#}(0)=z(0)$, $z^{\#}(T)=z(T)$. Moreover, if inequality (5.5) is strict, then $z$ is the unique minimizer. This completes the proof of Lemma 5.2.

The proof of Theorem 5.1 uses the following result.

Lemma 5.3. Let $q$ be a generalized incompressible flow that reaches the final configuration $\eta$ at time $T$ with finite kinetic energy. Assume that $B(z)=$ $b(z(0), z(T)$ ) holds q-almost everywhere on $\Omega$ (where $b$ and $B$ are defined by (5.7) and (5.8). Then $q$ is optimal.

Proof of Lemma 5.3. Since $\rho$ is bounded away from zero and the kinetic energy $E(q)$ is finite, $\iint\left\|z^{\prime}(t)\right\|^{2} d t q(d z)$ is also finite. It follows that $q$-almost every path $z$ belongs to $H^{1}[0, T]$ and, by Proposition 3.4,

$$
\begin{gathered}
V(z)=\rho(z(0)) \int U(t, z(t)) d t, \\
P(z)=\int p(t, z(t)) d t
\end{gathered}
$$

define $q$-integrable functions on $\Omega$. Moreover, (by taking $\rho=1$ in Proposition 3.4) we get

$$
\int P(z) q(d z)=\iint p(t, x) d t d x .
$$

These properties also hold for any other generalized incompressible flow $q^{\#}$ that has a finite kinetic energy. For such a flow, let us compute the integral of $B(z)$ (defined by (5.7)). We have $B(z)=e(z)-V(z)-P(z)$, where $e(z)=$ $\rho(z(0)) \int \frac{1}{2}\left\|z^{\prime}(t)\right\|^{2} d t$. We get

$$
\begin{aligned}
\int B(z) q^{\#}(d z) & =\int(e(z)-V(z)) q^{\#}(d z)-\int P(z) q^{\#}(d z) \\
& \quad\left(\text { since } e, V, \text { and } P \text { are } q^{\#}\right. \text {-integrable) } \\
& =\int(e(z)-V(z)) q^{\#}(d z)-\iint p(t, x) d t d x \quad(\text { by }(5.20)) \\
& =A\left(q^{\#}\right)-\iint p(t, x) d t d x \quad \text { (by definition (3.5) of the Action). }
\end{aligned}
$$

We deduce

$$
A\left(q^{\#}\right)-A(q)=\int B(z) q^{\#}(d z)-\int B(z) q(d z),
$$

since the pressure term is the same for $q$ and $q^{\#}$. To prove that $q$ is optimal, it is enough to show $\int B(z) q^{\#}(d z) \geq \int B(z) q(d z)$. To do that, let us consider the auxiliary function $z \in \Omega \rightarrow b(z(0), z(T))$. We know that $B(z) \geq$ $b(z(0), z(T))$ holds everywhere on $\Omega$ (by (5.11)), while $B(z)=b(z(0), z(T))$ is true $q$-almost everywhere, by assumption. Thus, $z \in \Omega \rightarrow b(z(0), z(T))$ is $q$-integrable, and, therefore, $b(x, y)$ is $\eta(d x, d y)$ integrable, since $q$ reaches $\eta$ at time $T$ where $\eta$ is defined by $\int f(z(0), z(T)) q(d z)=\int f(x, y) \eta(d x, d y)$ 
for each $f$ in $C(X \times X)$. It follows that $z \in \Omega \rightarrow b(z(0), z(T))$ is also $q^{\#}$-integrable, since $q^{\#}$ also reaches $\eta$ at time $T$. Thus,

$$
\int b(z(0), z(T)) q^{\#}(d z)=\int b(z(0), z(T)) q(d z)=\iint b(x, y) \eta(d x, d y),
$$

and, consequently,

$$
\begin{aligned}
\int B(z) q^{\#}(d z) & \geq \int b(z(0), z(T)) q^{\#}(d z) \\
& =\int b(z(0), z(T)) q(d z)=\int B(z) q(d z),
\end{aligned}
$$

which completes the proof of Lemma 5.3.

Proof of Theorem 5.1. The first statement of Theorem 5.1 immediately follows from Lemmas 5.2 and 5.3. Under assumptions (5.1)-(5.5), the generalized flow $q$ is necessarily optimal. Let us now consider the case when inequality (5.5) is strict. From Lemma 5.2, it is easy to deduce that $q$ is deterministic. Indeed, let us consider two paths $z$ and $z^{\#}$ that satisfy $z(0)=z^{\#}(0)$ and $z(T)=z^{\#}(T)$. We obviously have $b\left(z^{\#}(0), z^{\#}(T)\right)=b(z(0), z(T))$ and, $q$-almost surely, $z$ and $z^{\#}$ belong to $H^{1}[0, T]$. Thus, by Lemma 5.2 (first statement), we deduce $B(z)=B\left(z^{\#}\right)$. By Lemma 5.2 (second statement) again it follows that $z=z^{\#}$, which precisely shows that $q$ is deterministic.

Let us now prove that $q$ is the unique optimal flow that reaches $\eta$ at time $T$. Let us consider another generalized incompressible flow $q^{\#}$ that has a finite kinetic energy, reaches $\eta$ at time $T$, and has the same Action as $q$. We have seen earlier that

$$
\begin{aligned}
A\left(q^{\#}\right)-A(q) & =\int B(z) q^{\#}(d z)-\int B(z) q(d z) \\
& =\int B(z) q^{\#}(d z)-\int b(z(0), z(T)) q(d z) \\
& =\int\{B(z)-b(z(0), z(T))\} q^{\#}(d z) .
\end{aligned}
$$

Since $B(z)-b(z(0), z(T)) \geq 0$ and $A\left(q^{\#}\right)-A(q)=0$, it follows that $B(z)=$ $b(z(0), z(T))$ holds $q^{\#}$-almost everywhere on $\Omega$. We know that the same property also holds $q$-almost everywhere on $\Omega$. From the last statement of Lemma 5.2, it follows that, for $q$-almost every $z$ and $q^{\#}$-almost every $z^{\#}$, $z(0)=z^{*}(0), z(T)=z^{\#}(T)$ imply $z=z^{\#}$. Since $q$ and $q^{\#}$ reach the same final configuration, we conclude that they must be equal.

This completes the proof of Theorem 5.1, except for the last statement about the case when equality (5.6) holds. There is a trivial example when (5.6) holds for which the minimizer is not unique. Let us consider the 2-dimensional motion inside the unit disk of a homogeneous fluid $(\rho(x)=$ cst $=1)$ without external forces. To reach at time $T=\pi$ the "mirror" final configuration 
$\eta(d x, d y)=\delta(y-h(x)) d x$, where $h\left(x_{1}, x_{2}\right)=\left(-x_{1},-x_{2}\right)$, there are two different classical solutions to the Euler equations corresponding to two rotational flows with opposite constant angular velocities

$$
\begin{array}{r}
g_{\varepsilon}\left(t, x_{1}, x_{2}\right)=\left(x_{1} \cos (\varepsilon t)+x_{2} \sin (\varepsilon t),-x_{1} \sin (\varepsilon t)+x_{2} \cos (\varepsilon t)\right), \\
0 \leq t \leq \pi, \varepsilon= \pm 1 .
\end{array}
$$

In both cases, the pressure field is $p(t, x)=\frac{1}{2}\|x\|^{2}$, and thus, $R=1$. Since $\alpha=1$ and $C=0$, equality (5.6) becomes $R T^{2}=\pi^{2}$ and, therefore, is trivially satisfied.

There are also cases when (5.6) holds for which solutions to the shortest path problem are not deterministic. This will be discussed in $\S 6$.

\section{EXPLICIT EXAMPLES OF NONDETERMINISTIC GENERALIZED SOLUTIONS TO THE EULER EQUATIONS}

In this section, explicit examples of solutions to the generalized shortest path problem are obtained in the case of homogeneous $(\rho=1)$ fluids without external forces $(U=0)$. These solutions are of the form considered in $\S 5$ and Theorem 5.1. The generalized incompressibility condition is satisfied, their kinetic energy is finite, and there exists a smooth pressure field $p$ such that each trajectory $z$ satisfies the dynamical equation $z^{\prime \prime}=-\operatorname{grad} p$. Therefore, they satisfy the Least Action Principle in the sense that they are solutions to the generalized shortest path problem, provided that the final time $T$ is not too large. Since the trajectories are not deterministic in general, these solutions can be considered as probabilistic generalized solutions to the Euler equations. In each case, the corresponding velocity field is a stationary measure-valued solution in the sense of DiPerna and Majda. The first step to get these explicit solutions is

Proposition 6.1. Let $p$ be a pressure field given in $W^{2, \infty}\left(\mathbf{R}^{d}\right)$ and $G$ the global flow map

$$
(t, x, v) \in \mathbf{R} \times \mathbf{R}^{d} \times \mathbf{R}^{d} \rightarrow(X(t, x, v), V(t, x, v)) \in \mathbf{R}^{d} \times \mathbf{R}^{d},
$$

corresponding to the ordinary differential system in $\mathbf{R}^{2 d}$,

$$
x^{\prime}=v, \quad v^{\prime}=-\operatorname{grad} p(x) .
$$

Assume that, for some real $c, X=\left\{x \in \mathbf{R}^{d} ; p(x) \leq c\right\}$ defines a smooth compact subset of $\mathbf{R}^{d}$ of Lebesgue measure equal to 1 . Then $K=\{(x, v) ; p(x)+$ $\left.\frac{1}{2}\|v\|^{2} \leq c\right\}$ defines a compact subset of $\mathbf{R}^{d} \times \mathbf{R}^{d}$, invariant under $G$, and its $x$-projection is $X$.

Let $\mu(d x, d v)$ be a probability measure on $K$, invariant under $G$, i.e.,

$$
\iint f(X(t, x, v), V(t, x, v)) \mu(d x, d y)=\iint f(x, v) \mu(d x, d v),
$$


for any time $t$ and $f$ in $C(K)$. Then $\mu(d x, d v)$ always is a stationary measure-valued solution to the Euler equations [6]. Given $T>0$, the generalized flow $q$ defined by

$$
\begin{aligned}
\int F(z) q(d z)=\iint F[t & \rightarrow X(t, x, v)] \mu(d x, d v), \\
& \text { for each } F \text { in } C_{\mathrm{fin}}(\Omega), \Omega=X^{[0, T]},
\end{aligned}
$$

is incompressible on $X$ if and only if

$$
\text { for any } f \text { in } C(X), \iint f(x) \mu(d x, d v)=\int_{X} f(x) d x .
$$

Moreover, if $D^{2} p(x) \leq \pi^{2} / T^{2}$ holds a.e. on $X$, then $q$ solves the shortest path problem for the final configuration $\eta$ defined by

$$
\iint_{X \times X} f(x, y) \eta(d x, d y)=\iint f(x, X(T, x, v)) \mu(d x, d v),
$$

for any $f$ in $C(X \times X)$.

Remark. It is natural to consider $\mu(d x, d v)$ as the (time independent) velocity field associated with $q$,

$$
\begin{aligned}
\int f\left(z(t), z^{\prime}(t)\right) q(d z) & =\iint f(X(t, x, v), V(t, x, v)) \mu(d x, d v) \\
& \quad \text { by definition }(6.3)) \\
& =\iint f(x, v) \mu(d x, d v) \quad \text { (by property (6.2)). }
\end{aligned}
$$

Proof of Proposition 6.1. Since $p$ belongs to $W^{2, \infty}\left(\mathbf{R}^{d}\right)$, then $(x, v) \rightarrow$ $(v,-\operatorname{grad} p(x))$ defines a Lipschitz continuous vector field on $\mathbf{R}^{d} \times \mathbf{R}^{d}$ and the flow map $G$ is globally defined on $\mathbf{R} \times \mathbf{R}^{d} \times \mathbf{R}^{d}$. Since $H(x, v)=p(x)+\frac{1}{2}\|v\|^{2}$ is constant along each trajectory, $K$ is invariant under $G$; it is also easy to check that $K$ is compact and $X$ is the $x$-projection of $K$.

Let us first prove that $\mu(d x, d v)$ is a time independent solution to the Euler equations in the sense of DiPerna and Majda. From the invariance property (6.2), one deduces for any time $t$ and any smooth bounded function $f$ defined on $X$

$$
\begin{gathered}
\iint f(X(t, x, v)) \mu(d x, d v)=\iint f(x) \mu(d x, d v), \\
\iint V(t, x, v) f(X(t, x, v)) \mu(d x, d v)=\iint v f(x) \mu(d x, d v) .
\end{gathered}
$$

Since $(X(t, x, v), V(t, x, v))$ is the solution to $x^{\prime}=v, v^{\prime}=-\operatorname{grad} p(x)$, by expanding these equalities with respect to $t$ about 0 , we deduce

$$
\begin{gathered}
\iint v \cdot \operatorname{grad} f(x) \mu(d x, d v)=0, \\
\iint[v(v \cdot \operatorname{grad} f(x))-\operatorname{grad} p(x) f(x)] \mu(d x, d v)=0,
\end{gathered}
$$


which means that $\mu$ is a time independent measure-valued solution to the Euler equations [6]. Let us now consider the generalized flow $q$ defined by (6.3). This flow is well defined on $X$ since for $\mu$-almost every $(x, v)$ in $\mathbf{R}^{d} \times \mathbf{R}^{d}$, the curve $t \rightarrow X(t, x, v)$ is valued in $X$ (indeed, $G$ is invariant under $G$, and $X$ is the $x$-projection of $K$ ).

The incompressibility condition means $\int f(z(t)) q(d z)=\int f(x) d x$, for any $t$ and $f$ in $C(X)$. Since, from definition (6.3) and property (6.2),

$$
\int f(z(t)) q(d z)=\iint f(X(t, x, v)) \mu(d x, d y)=\iint f(x) \mu(d x, d v),
$$

it follows that $q$ is a generalized incompressible flow on $X$ if and only if condition (6.4) holds. The kinetic energy of $q$, which is exactly equal to

$$
\frac{1}{2} \iint\|v\|^{2} \mu(d x, d v),
$$

if finite. Finally, since $q$-almost every path $z$ in $\Omega$ is of the form $z(t)=$ $X(t, x, v)$ and is a solution to the ordinary differential equation $z^{\prime \prime}=\operatorname{grad} p(z)$, it follows from Theorem 5.1 that $q$ is a solution to the generalized shortest path problem for the final configuration $\eta$ given by (6.5), which completes the proof.

Among the invariant positive measures for the Hamiltonian system $x^{\prime}=v$, $v^{\prime}=-\operatorname{grad} p$, there is obviously $d x d v$ (by Liouville's theorem) and also any measure $\mu$ of the form

$$
\mu(d x, d v)=\lambda(H(x, v)) d x d v, \quad H(x, v)=\frac{1}{2}\|v\|^{2}+p(x),
$$

where $\lambda$ is an arbitrary positive function (indeed, $H$ is constant along the trajectories). For these measures, which are uniform on each level set of $H$, we have

Proposition 6.2. Under the same conditions as Proposition 6.1, let $\mu$ be a probability measure of the form (6.9). Then, although $\mu$ always is a measure-valued solution to the Euler equations in the sense of DiPerna and Majda, the corresponding generalized flow q defined by (6.3) is incompressible (and, therefore, solves the generalized shortest path problem) only in the following cases: (i) $\lambda(t)=\mathrm{Cst} \cdot \delta(t-c)$, when $d=2$; (ii) $\lambda(t)=\mathrm{Cst} \cdot(c-t)^{-1 / 2}$, when $d=1$; (iii) and never when $d=3$.

Proof of Proposition 6.2. If $\mu$ is of the form (6.9), and, more generally, if $\mu$ is a measure depending only on $x$ and $\|v\|$ (which means that, at each point $x$, the velocity is isotropically distributed), then it is automatically a measure solution in the sense of DiPerna and Majda.

Indeed, if $\mu(d x, d v)=\sigma(x,\|v\|) d x d v$, we get

$$
\begin{gathered}
\iint v_{i} f(x) \mu(d x, d v)=\iint v_{i} \sigma(x,\|v\|) f(x) d x d v=0, \\
\iint v_{i} v_{j} f(x) \mu(d x, d v)=\iint v_{i} v_{j} \sigma(x,\|v\|) f(x) d x d v=\delta_{i j} \int \phi(x) f(x) d x,
\end{gathered}
$$


where $\phi(x)=\frac{1}{d} \int\|v\|^{2} \sigma(x,\|v\|) d v$. Then it is easy to check that $\mu$ is a measure-valued solution to the Euler equations.

Conversely, the requirement that the associated generalized flow $q$ is incompressible (and, therefore, satisfies the generalized Least Action Principle, in the sense that it is a solution to the shortest path problem when $T$ is not too large) is considerably more restrictive. According to Proposition 6.1, this happens if and only if property (6.4) holds. Since $\mu(d x, d v)=\lambda\left(\frac{1}{2}\|v\|^{2}+p(x)\right) d x d v$, this means exactly that $\int \lambda\left(\frac{1}{2}\|v\|^{2}+p(x)\right) d v=1$ if $x$ belongs to $X, 0$ otherwise, and, therefore, is equivalent to $\int \lambda\left(\frac{1}{2}\|v\|^{2}+s\right) d v=1$ if $s \leq c, 0$ if $s>c$, since $X=\left\{x \in \mathbf{R}^{d}, p(x) \leq c\right\}$. Finally, by using polar coordinates, we see that $\lambda$ must satisfy the following classical integral equation

$$
\begin{aligned}
C_{d} \int_{r>0} \lambda(r+s) r^{d / 2-1} d r & =1, & & \text { if } s \leq c, \\
& =0, & & \text { if } s>c,
\end{aligned}
$$

where $C_{d}$ is a numerical constant depending on the space dimension. This equation has no positive solution when $d=3$. The unique solution is (up to a multiplicative constant depending on $d$ )

$$
\lambda(r)=\delta(r-c) \quad \text { when } d=2
$$

(then $\lambda$ is not a function and (6.9) is more a convenient notation than a rigorous definition).

$$
\begin{aligned}
\lambda(r) & =(c-r)^{-1 / 2}, \quad \text { if } r<c, \\
& =0, \quad \text { if } r \geq c, \quad \text { when } d=1 .
\end{aligned}
$$

This completes the proof of Proposition 6.2.

This result allows us to construct a large family of generalized solutions to the Euler equations in dimension 2 and 1! Let us consider the simplest examples when

$$
p(x)=\frac{1}{2}\|x\|^{2}, \quad X=\left\{x \in \mathbf{R}^{d},\|x\| \leq 1\right\}, \quad d=1 \text { or } 2 .
$$

Then the flow map $G$ is trivially given by

$$
X(t, x, v)=x \cos t+v \sin t, \quad V(t, x, v)=-x \sin t+v \cos t .
$$

Following Proposition 6.2, after some elementary computations, we find a 2dimensional generalized solution $q$ defined by

$$
\int F(z) q(d z)=\pi^{-2} \iint F[t \rightarrow x \cos t+v \sin t] \delta\left(\|x\|^{2}+\|v\|^{2}-1\right) d x d v,
$$

for each $F$ in $C_{\text {fin }}(\Omega)$, 
that is

$$
\begin{aligned}
& \int F(z) q(d z) \\
& \quad=\pi^{-2} \int_{\|x\|<1} \int_{0<\theta<\pi} F\left[t \rightarrow x \cos t+\left(1-\|x\|^{2}\right)^{1 / 2}(\cos \theta, \sin \theta) \sin t\right] d \theta d x .
\end{aligned}
$$

The corresponding 1-dimensional solution is obtained in a similar way:

$$
\begin{aligned}
& \int F(z) q(d z) \\
& \quad=(2 \pi)^{-1} \int_{|x|<1} \int_{0<\theta<\pi} F\left[t \rightarrow x \cos t+\left(1-x^{2}\right)^{1 / 2} \cos \theta \sin t\right] d \theta d x .
\end{aligned}
$$

When $T=\pi$, in both cases the final configuration $\eta$ is deterministic and given by

$$
\eta(d x, d y)=\delta(y-h(x)) d x, \quad \text { where } h(x)=-x .
$$

Moreover, the pressure field identically satisfies $D^{2} p(x)=\pi^{2} / T^{2} I$ (where $I$ is the identity). Since $T=\pi$ exactly corresponds to the limit case of Theorem 5.1 (when equality (5.6) holds), it follows that the generalized flows defined by (6.16) and (6.17) are solutions to the shortest path problem. Notice that they are not deterministic, although the final configuration is deterministic! Thus, the last statement of Theorem 5.1 is now entirely justified.

In dimension 2, the final configuration $h(x)=-x$ is classical (it is a smooth volume and orientation preserving map), and the shortest path problem has two trivial classical solutions, already considered in $\S 5$, the two rotational flows with opposite constant angular velocities:

$$
\begin{aligned}
g_{\varepsilon}\left(t, x_{1}, x_{2}\right)=\left(x_{1} \cos (\varepsilon t)+x_{2} \sin (\varepsilon t),-x_{1} \sin (\varepsilon t)\right. & \left.+x_{2} \cos (\varepsilon t)\right) \\
0 & \leq t \leq \pi, \varepsilon= \pm 1 .
\end{aligned}
$$

Thus, (6.16) defines another, highly nonclassical, solution to the same (generalized) shortest path problem! It can easily be checked that the three different solutions have the same kinetic energy and, more surprisingly, the same pressure field $p(x)=\frac{1}{2}\|x\|^{2}$. In contrast with the two classical flows, the probabilistic one has a zero mean velocity field, while the pressure is in exact balance with the inertial tensor

$$
E\left[v_{i}\right]=0, \quad E\left[v_{i} v_{j}\right]=\delta_{i j}\left(\frac{1}{2}-p(x)\right),
$$
where $E$ denotes the expected value.

In the 1-dimensional case, the final configuration is still deterministic but not classical (indeed $h(x)=-x$ is volume preserving but orientation reversing). Therefore, there is no classical solution to the shortest path problem. Further- 
more, one can prove

Proposition 6.3. Let $X=[-1,+1]$. For the final configuration $h(x)=-x$ and the final time $T=\pi$, the generalized shortest path problem has a unique solution $q$ defined by (6.17).

Proof of Proposition 6.3. We already know that (6.17) defines a solution $q$ to the shortest path problem. Let us prove that this solution is unique. Because $q$-almost every path $z$ is a solution to $z^{\prime \prime}=-z$ and satisfies $\int\left\|z^{\prime}(t)\right\|^{2} d t=$ $\int\|z(t)\|^{2} d t$ (where the integral is performed over $[0, \pi]$ ), it follows that

$$
\begin{aligned}
E(q) & =\iint \frac{1}{2}\left\|z^{\prime}(t)\right\|^{2} d t q(d z)=\iint \frac{1}{2}\|z(t)\|^{2} d t q(d z) \\
& =\iint \frac{1}{2}\|x\|^{2} d t d x=\pi \int \frac{1}{2}\|x\|^{2} d x
\end{aligned}
$$

(since $q$ is incompressible). Therefore, any optimal flow $q^{*}$ must satisfy

$$
\begin{aligned}
\iint \frac{1}{2}\left\|z^{\prime}(t)\right\|^{2} d t q^{*}(d z) & =E(q)=\pi \int \frac{1}{2}\|x\|^{2} d x \\
& =\iint \frac{1}{2}\|x\|^{2} d t d t=\iint \frac{1}{2}\|z(t)\|^{2} d t q^{*}(d z)
\end{aligned}
$$

(indeed, $q^{*}$ also is incompressible). Since the final configuration at time $T=$ $\pi$ is $h(x)=-x$, we get $z(\pi)=-z(0)$ for $q^{*}$-almost every path $z$. By Poincaré's inequality, $z(\pi)=-z(0)$ implies $\int\left\|z^{\prime}(t)\right\|^{2} d t \geq \int\|z(t)\|^{2} d t$ (where the integral is performed over $[0, \pi])$ and the corresponding equality holds if and only if $z$ is of the form $z(t)=x \cos t+v \sin t$, for some real numbers $x$ and $v$. Because $z$ maps $[0, \pi]$ into $[-1,+1],(x, v)$ must belong to the unit disk. Then it is not hard to deduce that there exists a probability measure $\mu(d x, d v)$ supported by the unit disk such that $q^{*}$ can be defined by (6.21)

$\int F(z) q^{*}(d z)=\iint F[t \rightarrow x \cos t+v \sin t] \mu(d x, d v), \quad$ for each $F$ in $C_{\text {fin }}(\Omega)$.

Since $q^{*}$ must be incompressible, $\mu(d x, d v)$ must satisfy

$$
\begin{aligned}
& \int f(x \cos t+v \sin t) \mu(d x, d v)=\int f(x) d x \\
& \text { for any } f \text { in } C[-1,+1] \text { and } t \text { in }[0, \pi]
\end{aligned}
$$

(here $d x$ denotes the Lebesgue measure multiplied by $\frac{1}{2}$, in order to be a probability measure on $[-1,+1])$. In particular, $(6.22)$ holds for any $f$ of the form $f(x)=\exp (i x \xi)$. Then it can be easily seen that the Fourier transform of $\mu(d x, d v)$ (which is a bounded continuous function defined on $\mathbf{R}^{2}$ ) is completely determined (notice that this assertion would be wrong in the 2dimensional case when $X$ is the disk!). Thus, $\mu$ is unique and, consequently, $q^{*}$ also is unique. This completes the proof of Proposition 6.3. 


\section{APPENDIX. A FORMAL LINK WITH THE MEASURE-VALUED SOLUTIONS IN THE SENSE OF DiPERNA AND MAJDA}

In this appendix, it is shown that we can formally associate to any optimal generalized flow a generalized velocity field that (formally) solves the Euler equations in the sense of DiPerna and Majda. For simplicity, it is assumed that $X$ is the closure of a smooth bounded open set in $\mathbf{R}^{d}$, the fluid is homogeneous $(\rho=1)$, and there is no external forces $(U=0)$.

Let $q$ be an optimal generalized incompressible flow. Let us introduce the probability measure $\mu(d t, d x, d v)$ on $[0, T] \times X \times \mathbf{R}^{d}$, formally defined by

$$
\iiint f(t, x, v) \mu(d t, d x, d v)=T^{-1} \iint f\left(t, z(t), z^{\prime}(t)\right) d t q(d z),
$$

for any suitable $f$ defined on $[0, T] \times X \times \mathbf{R}^{d}$ (it is not easy, for several reasons, to justify this definition). $\mu$ can be considered, as a generalized velocity field has a finite kinetic energy

$$
\iint \frac{1}{2}\|v\|^{2} \mu(d t, d x, d v)=E(q)<+\infty,
$$

and is a solution to the Euler equations in the following generalized sense (cf. [6]):

$$
\iiint\left[v \cdot w(x) f^{\prime}(t)+v(D w(x) \cdot v) f(t)\right] \mu(d t, d x, d v)=0,
$$

for any smooth real function $f$ compactly supported in $] 0, T[$ and any smooth vector field $w$ on $X$ such that $\operatorname{div} w=0$ inside $X, w \cdot n=0$ along $\partial X$ ( $n=$ outward normal).

Let us give a formal proof of this claim. Because of the properties of $w$, there is a smooth classical volume preserving flow map $\gamma$, $(t, x) \in \mathbf{R} \times X \rightarrow \gamma(t, x) \in X, \quad$ such that $\gamma(0, x)=x, \partial_{t} \gamma(t, x)=w(\gamma(t, x))$.

For $\varepsilon$ small, let us consider the modified generalized flow $q_{\varepsilon}$ defined by

$$
\int_{\Omega} F(z) q_{\varepsilon}(d z)=\int_{\Omega} F[t \rightarrow \gamma(\varepsilon f(t), z(t))] q(d z), \text { for each } F \text { in } C_{\mathrm{fin}}(\Omega) .
$$

It is easy to check that $q_{\varepsilon}$ is incompressible. Indeed, for each $f$ in $C(X)$ and $t$ in $[0, T]$,

$$
\begin{aligned}
\int f(z(t)) q_{\varepsilon}(d z) & =\int f(\gamma(\varepsilon f(t), z(t))) q(d z)=\int f(\gamma(\varepsilon f(t), x)) d x \\
& =\int f(x) d x \quad \text { (since } \gamma \text { is volume preserving). }
\end{aligned}
$$

Let us now compare the kinetic energies of $q$ and $q_{\varepsilon}$. A formal computation leads to

$$
\begin{gathered}
\gamma(\varepsilon f(t), z(t))=z(t)+\varepsilon f(t) w(z(t))+0\left(\varepsilon^{2}\right), \\
\partial_{t}[\gamma(\varepsilon f(t), z(t))]=z^{\prime}(t)+\varepsilon \partial_{t}[f(t) w(z(t))]+0\left(\varepsilon^{2}\right) .
\end{gathered}
$$




\section{It follows that}

$$
\begin{aligned}
E\left(q_{\varepsilon}\right) & -E(q)=\iint\left[\frac{1}{2}\left\|\partial_{t}[\gamma(\varepsilon f(t), z(t))]\right\|^{2}-\frac{1}{2}\left\|z^{\prime}(t)\right\|^{2}\right] d t q(d z) \\
& =\varepsilon \iint z^{\prime}(t) \cdot \partial_{t}[f(t) w(z(t))] d t q(d z)+0\left(\varepsilon^{2}\right) \\
& =\varepsilon \iint\left[z^{\prime}(t) \cdot w(z(t)) f^{\prime}(t)+z^{\prime}(t)\left(D w(z(t)) \cdot z^{\prime}(t)\right)\right] d t q(d z)+0\left(\varepsilon^{2}\right) \\
& =\varepsilon \iiint\left[v \cdot w(x) f^{\prime}(t)+v(D w(x) \cdot v) f(t)\right] \mu(d t, d x, d v)+0\left(\varepsilon^{2}\right),
\end{aligned}
$$

which shows that $\iiint\left[v \cdot w(x) f^{\prime}(t)+v(D w(x) \cdot v) f(t)\right] \mu(d t, d x, d v)$ must vanish for each suitable $w$.

\section{REFERENCES}

1. V. I. Arnol'd, Méthodes mathématiques de la mécanique classique, Mir, Moscow, 1976.

2. V. I. Arnol'd and A. Avez, Problèmes ergodiques de la mécanique classique, Gauthier-Villars, Paris, 1967.

3. N. Bourbaki, Eléments de mathématique, Integration (Chapitre 4), Hermann, Paris, 1965.

4. Y. Brenier, Décomposition polaire et réarrangement monotone des champs de vecteurs, $\mathrm{C}$. $\mathbf{R}$. Acad. Sci. Paris Sér. I Math. 305 (1987), 805-808.

5. R. DiPerna, Measure-valued solutions of conservation laws, Arch. Rational Mech. Anal. 88 (1985), 223-270.

6. R. DiPerna and A. Majda, Oscillations and concentrations in weak solutions of the incompressible fluid equations, Comm. Math. Phys. 108 (1987), 667-689.

7. D. Ebin and J. Marsden, Groups of diffeomorphisms and the motion of an incompressible fluid, Ann. of Math. (2) 92 (1970), 102-163.

8. I. Ekeland and R. Temam, Analyse convexe et problemes variationnels, Dunod, Paris, 1974.

9. S. T. Rachev, The Monge-Kantorovitch mass transference problem, Theory Probab. Appl. (29) (4) (1985), 647-676.

10. M. Reed and B. Simon, Methods of modern mathematical physics, II, Academic Press, New York, 1978.

11. L. Tartar, The compensated compactness method applied to systems of conservation laws, Systems of nonlinear PDE (J. Ball, ed.), NATO ASI Series, Reidel, Dordrecht, 1983.

12. L. C. Young, Lectures on the calculus of variations and optimal control theory, Saunders, Philadelphia and London, 1969.

ABstract. The link between the Euler equations of perfect incompressible flows and the Least Action Principle has been known for a long time [1]. Solutions can be considered as geodesic curves along the manifold of volume preserving mappings. Here the "shortest path problem" is investigated. Given two different volume preserving mappings at two different times, find, for the intermediate times, an incompressible flow map that minimizes the kinetic energy (or, more generally, the Action). In its classical formulation, this problem has been solved [7] only when the two different mappings are sufficiently close in some very strong sense. In this paper, a new framework is introduced, where generalized flows are defined, in the spirit of $L$. C. Young, as probability measures on the set of all possible trajectories in the physical space. Then the minimization problem is generalized as the "continuous linear programming" problem that is much easier to handle. The existence problem is completcly solved in 
the case of the $d$-dimensional torus. It is also shown that under natural restrictions a classical solution to the Euler equations is the unique optimal flow in the generalized framework. Finally, a link is established with the concept of measure-valued solutions to the Euler equations [6], and an example is provided where the unique generalized solution can be explicitly computed and turns out to be genuinely probabilistic.

INRIA BP 105, 78153 Le Chesnay Cedex, France 